\title{
A low-cost plasmonic sensing device for on-site detection of diclofenac molecules
}

\author{
Nadja Steinke ${ }^{1}$, Roland Wuchrer ${ }^{1}$, Gerald Gerlach ${ }^{2}$, Thomas Härtling ${ }^{1,2}$ \\ ${ }_{1}^{1}$ Fraunhofer Institute for Ceramic Technologies and Systems IKTS, Maria-Reiche-Straße 2, D-01109, \\ Dresden, Germany, \\ ${ }^{2}$ Technische Universität Dresden, Institute of Solid State Electronics, Mommsenstraße 15, D-01069, \\ Dresden, Germany \\ nadja.steinke@ikts.fraunhofer.de
}

\begin{abstract}
:
In the field of environmental and life sciences surface plasmon resonance (SPR) sensors are wellestablished. To circumvent the current limitation of SPR sensors to applications in the laboratory environment studies on a low-cost nanostructured sensor substrate fabricated by nanoimprint lithography were conducted. The nanostructured sensor substrate is the core element for the development of a SPR-based on-site biosensor system including a novel photocurrent-based read-out approach. For this purpose, diclofenac molecules were successfully immobilized on the sensor substrates and a specific binding of an anti-diclofenac antibody with subsequent regeneration can be observed in form of a shift in the optical transmission spectra. The results show that our functionalized transducer element based on SPR is well-suited for the detection of diclofenac.
\end{abstract}

Key words: Optical biosensor, Surface plasmon resonance, Nanoimprint lithography, Diclofenac, Anti-diclofenac antibody.

\section{Introduction}

The detection of hormones and pharmaceuticals at trace concentrations is of growing interest due to their presence in the aquatic environment (in the range of $\mathrm{ng} / \mathrm{L}$ to $\mu \mathrm{g} / \mathrm{L}$ ) and potential toxic effects on nature and wildlife. Remarkably high concentrations at the effluent of waste water treatment plants are measured from the nonsteroidal anti-inflammatory drug diclofenac [1]. Hence, it can be considered as a guidance substance for the presence of pharmaceutical residues after water treatment processes.

Current detection methods, e.g. GC-MS or LCMS/MS are realized by representative sampling, which is time-consuming and costly. To overcome these problems the monitoring of pharmaceuticals needs to be done on-site, e.g. directly at the effluent of water treatment plants. This leads to a demand of small, sensitive and robust biosensors allowing continuous monitoring of molecule concentrations in complex matrices.

\section{Experiments and results}

Particularly suitable for this purpose are surface plasmon resonance (SPR) sensors allowing a label-free and sensitive detection of bio- molecules in real-time. By means of an indirect, competitive assay with a monoclonal antidiclofenac antibody [2] we were able to detect diclofenac concentrations in the range of less than $5 \mu \mathrm{g} / \mathrm{L}$ with a laboratory SPR system (capitalis technology, Berlin, Germany). However, this system is designed for use in laboratory environment only, which is why we extended our study and furthermore present the development of a plasmonic sensor system for the fast on-site detection of diclofenac molecules.

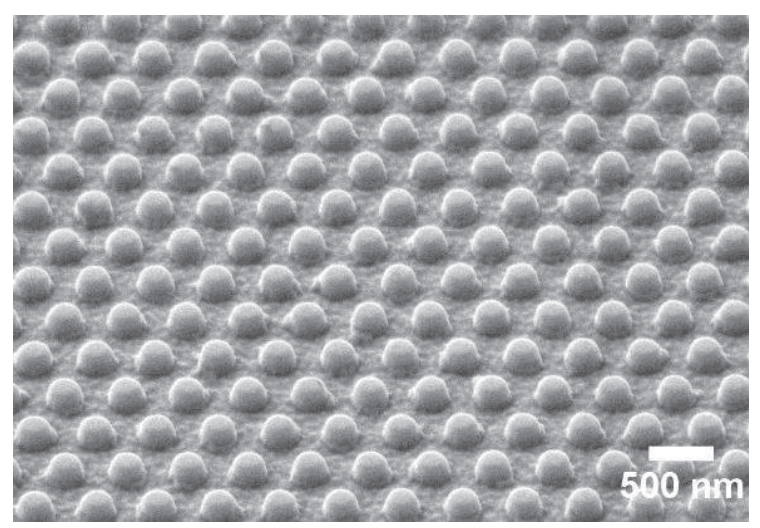

Fig. 1. SEM image of a nanostructured sensor substrate fabricated by nanoimprint lithography. 
At this, a nanostructured gold surface fabricated by nanoimprint lithography plays a key role and serves as plasmonic transducer [3]. The nanostructure in form of an array of pillars was imprinted by means of the $\mu$ ContactPrinter 4.1 (GeSiM mbH, Großerkmannsdorf, Deutschland). Figure 1 depicts a SEM image (Zeiss Nvision 40, Zeiss, Oberkochen, Germany) of a nanostructured sensor substrate.

The transducer surface is coated with a heterobifunctional polyethylene glycol (Rapp Polymere, Tübingen, Germany) at the upper end of which diclofenac is bonded covalently [3]. In this contribution we present transmission spectra (Maya2000 Pro, Ocean Optics, Largo, USA) of the pre-functionalized nanostructured sensor substrates before and after incubation with an anti-diclofenac antibody (Figure 2). By means of a tape-based microfluidic cell in combination with syringe pumps (GeSiM $\mathrm{mbH}$, Großerkmannsdorf, Deutschland) a continuous fluid flow and reproducible measurements were realized.

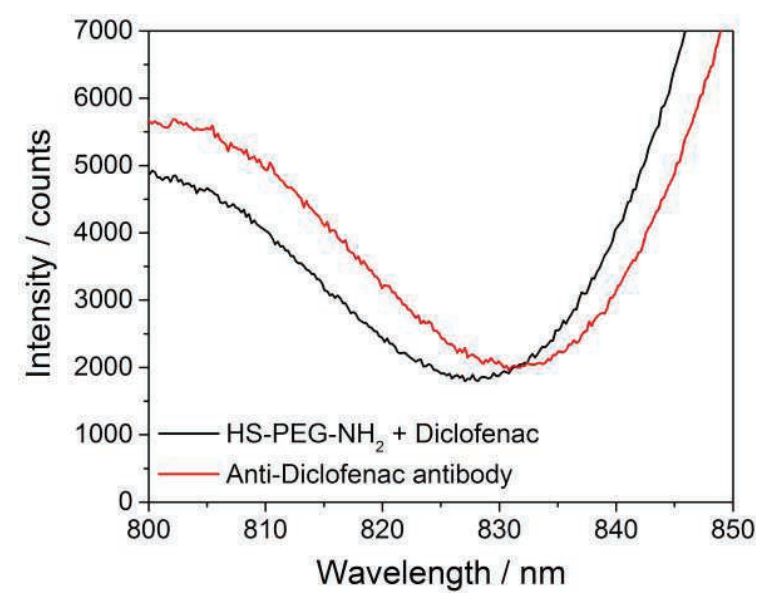

Fig. 2. Transmission spectra of a diclofenacfunctionalized sensor substrate before and after incubation with an anti-diclofenac antibody.

The specific binding of the anti-diclofenac antibody and its subsequent regeneration can be observed in form of a surface plasmon resonance shift in the optical transmission spectrum.

To further minimize the optical unit and sensor size a compact and robust optical transmittance configuration was set up additionally, which relies on a novel photocurrent-based read-out approach [4]. Initial experiments with a focus on the applied surface functionalization to demonstrate the sensitivity of the holistic plasmonic sensor system for the detection of diclofenac molecules in the low $\mu \mathrm{g} / \mathrm{L}$ range will be presented.

\section{Conclusion}

In this contribution we report on the successful functionalization of diclofenac molecules on a nanostructured sensor substrate. Besides the specific binding of an anti-diclofenac antibody and subsequent regeneration are promising results to demonstrate the sufficient sensitivity of our holistic plasmonic sensor system to detect diclofenac molecules below a concentration of $10 \mu \mathrm{g} / \mathrm{L}$.

\section{Acknowledgments}

The authors thank Rüdiger Rosenkranz for SEM imaging. This work was funded by the DFG research training group "Nano- and Biotechnologies for Packaging of Electronic Systems" DFG 1401/2 and the BMBF (German Federal Ministry of Education and Research) as part of the photonics research Germany funding program (grant number 13N13767).

\section{References}

[1] A.C. Mehinto, E.M. Hill, C.R. Tyler, Uptake and biological effects of environmentally relevant concentrations of the nonsteroidal antiinflammatory pharmaceutical diclofenac in rainbow trout (Oncorhynchusmykiss), Environ. Sci. Technol. 44, 2176-2182 (2010); doi:10.1021/es903702m

[2] M. Huebner, E. Weber, R. Niessner, S. Boujday, D. Knopp, Rapid analysis of diclofenac in freshwater and wastewater by a monoclonal antibody-based highly sensitive ELISA, Anal. Bioanal. Chem. 407, 8873-8882 (2015); doi: 10.1007/s00216-015-9048-9

[3] N. Steinke, M. Rio, R. Wuchrer, C. Schuster, E. Ljasenko, D. Knopp, G. Gerlach, T. Härtling, Detection of diclofenac molecules by planar and nanostructured plasmonic sensor substrates, Sens Actuator B Chem. 254, 749-754 (2018); doi: 10.1016/j.snb.2017.07.123

[4] R. Wuchrer, S. Amrehn, L. Liu, T. Wagner, T. Härtling, A compact readout platform for spectraloptical sensors, J. Sens. Sens. Syst. 5, 157-163 (2016); doi: 10.5194/jsss-5-157-2016 